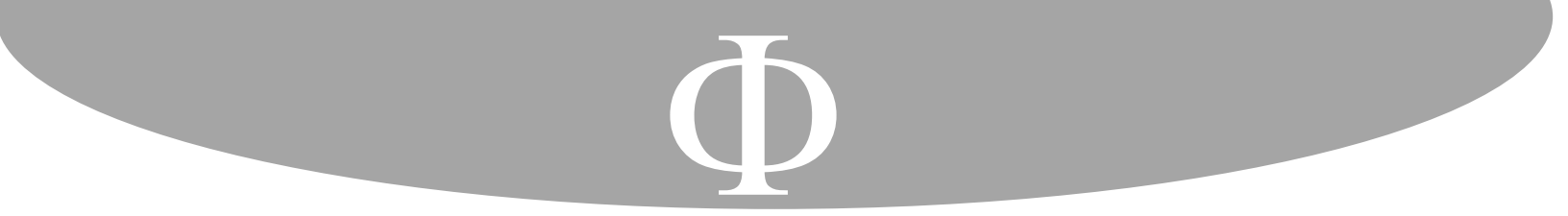

\title{
La Revelación y su relación con Cristo y la Trinidad según el planteo fenomenológico de Jean-Luc Marion en Givenness \& Revelation
}

Para citar este artículo: Roldán, Alberto F. «La Revelación y su relación con Cristo y la Trinidad según el planteo fenomenológico de Jean-Luc Marion en Givenness \& Revelation». Franciscanum 177, Vol. 64 (2022): 1-18.

\section{Resumen}

En el presente artículo el autor expone el tema de la Revelación y su relación con Cristo y la Trinidad en la perspectiva fenomenológica de Jean-Luc Marion. El centro de su análisis radica en la obra del filósofo francés Givenness \& Revelation. En cuanto al enfoque cristológico, Marion enfatiza a Cristo como ícono de Dios y, a partir de una exégesis fenomenológica de 1 Corintios 2, invita a una radical anamorfosis que permita contemplar a Cristo como centro de la sabiduría de Dios. En cuanto a la Trinidad, destaca los aportes de Karl Rahner y Karl Barth, profundizando en los padres capadocios y San Agustín, para enfatizar la función del Espíritu en el despliegue trinitario de Dios.

\section{Palabras clave}

Marion, Fenomenología, Revelación, Cristo, Trinidad.

The Revelation and its relationship with Christ and the Trinity according to the phenomenological approach of Jean-Luc Marion in Givenness \& Revelation

\begin{abstract}
In this article, the author expounds the theme of Revelation and its relationship with Christ and the Trinity in the phenomenological perspective of Jean-Luc Marion. The center of His analysis is the book of Marion Givenness \& Revelation. In relation to the Christological the emphasis of Marion is in Christ as icon of God and from a

\footnotetext{
* Doctor en teología por el Instituto Universitario Isedet (Buenos Aires), master en filosofía política (Universidad Nacional de Quilmes) y master en educación (Universidad del Salvador, Buenos Aires). Es director de posgrado del Instituto Teológico Fiet, profesor adjunto de la Universidad Adventista del Plata, Semisud, Lee University y South African Theological Seminary. Autor de unos treinta libros en el campo de la teología, la ética, la hermenéutica y la fenomenología, con traducciones al inglés y al portugués. Sus más recientes publicaciones son: Hermenéuticas y éticas (Oregon: Publicaciones Kerigma, 2021) y Dios y la narrativa de los tiempos. Tras las huellas del Apocalipsis en la literatura latinoamericana (Buenos Aires: Juanuno1 ediciones, 2021). Ha dictado cursos y conferencias en América Latina, Estados Unidos, España y Corea del Sur. ORCID: https://orcid.org/0000-0003-0353-9004. Contacto: roldan1967@ gmail.com.
} 


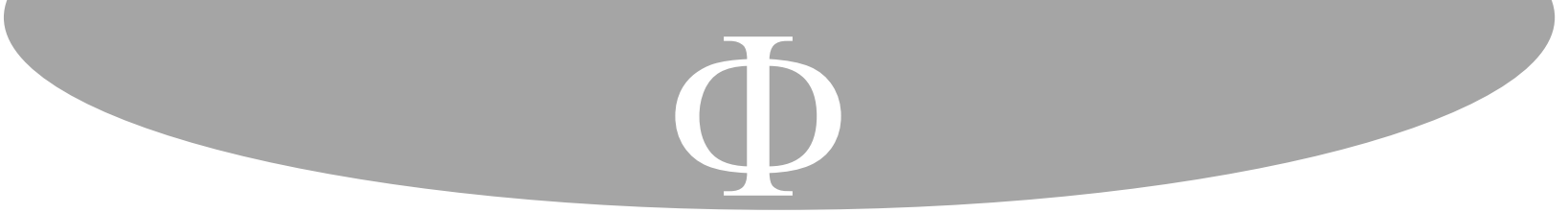

phenomenological exegesis of 1 Corinthians 2. He invites to a radical anamorphosis what allows to contemplate to Christ as a center of the wisdom of God. In relationship to the Trinity, Marion distinguish the contributions of Karl Rahner and Karl Barth, the Cappadocian Fathers and Saint Augustine with emphasis in the function of the Spirit in the Trinitarian deployment of God.

\section{Key words}

Marion, Phenomenology, Revelation, Christ, Trinity.

La única mirada y el único punto de vista que puede hacer que la infinita hipérbole de la caridad se dé a sí misma es la que se fundamenta en Cristo; la única mirada fenomenológicamente infinita, aún en nuestra carne ${ }^{1}$.

El Espíritu se impone a sí mismo como el fenoménico medio de acceso a la visión icónica del Padre en el Hijo como Jesús el Cristo, funcionado como director del despliegue trinitario de Dios $^{2}$. Jean-Luc Marion

E indiscutiblemente, grande es el misterio de la piedad: Dios fue manifestado en carne [ $\varepsilon \vee \sigma \alpha \rho \kappa \imath] .1$ Timoteo 3.16

Jean-Luc Marion es uno de los representantes más conspicuos de la fenomenología actual. Uno de sus aportes más importantes consiste en hacer del fenómeno saturado el paradigma de la fenomenicidad. Para el filósofo francés, los fenómenos saturados son aquellos cuya intuición sobrepasa o desborda al concepto. Básicamente hay cuatro fenómenos saturados a saber: el acontecimiento histórico, el ídolo, el cuerpo (la carne) y el rostro del prójimo. Pero a ellos se puede agregar otro fenómeno saturado -acaso el quintoque es el fenómeno de la revelación. En el presente trabajo, analizamos la Revelación en sentido teológico -para lo cual Marion siempre escribe con «R»- como concentrador de otros fenómenos saturados, su relación con Cristo y con la trinidad y la influencia de Barth, el aporte de los padres capadocios y San Agustín, destacando al final la función del Espíritu en el despliegue trinitario de Dios.

\section{La Revelación como concentrador de otros fenómenos saturados}

Marion dice:

\footnotetext{
${ }^{1}$ Jean-Luc Marion, Givenness \& Revelation, trad. Stephen E. Lewis (Oxford: University Press, 2016), 72. Este es el texto que tomamos como base para nuestra presente investigación. Existe libro posterior del mismo autor, titulado D'Ailleurs La Révelátion, Grasset, noviembre de 2020 que, dada su envergadura, se reserva para futuros trabajos sobre el tema.

2 Jean-Luc Marion, Givenness \& Revelation, 106
} 


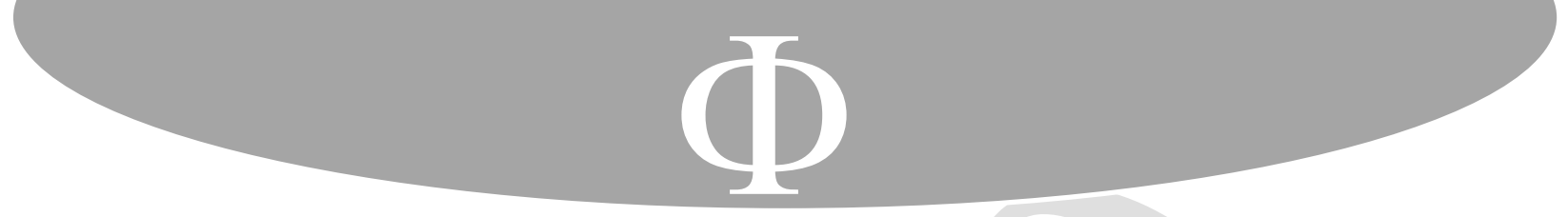

Se trata de la última variación posible de la fenomenicidad del fenómeno en tanto que dado -el fenómeno de revelación no solamente depende de la saturación (paradoja en general), sino que concentra en él los cuatro tipos de fenómenos saturados y se da a la vez como acontecimiento histórico, como ídolo, como carne y como ícono (rostro) ${ }^{3}$.

Para Marion, este quinto tipo, estrictamente, no añade nada nuevo a los cuatro anteriores, sino que la Revelación desborda de modo que satura la fenomenicidad como una saturación de la saturación, en otras palabras, una saturación plena. En ese contexto, Marion admite que la fenomenología tiene sus límites al enfrentarse a este fenómeno. Porque, explica:

La fenomenología no podría decidir si una revelación puede o no debe darse jamás, pero sí que puede (y únicamente la fenomenología puede) establecer que, en ese caso, un tal fenómeno de revelación debería tomar la figura de paradoja de paradojas: si tiene que haber revelación (y la fenomenología no tiene ninguna autoridad para decirlo), entonces esa revelación tomará, toma o ha tomado la figura de paradoja de paradojas, siguiendo una ley de esencia de la fenomenicidad ${ }^{4}$.

En otro texto, Marion cita a Marlène Zareder que, de modo más rotundo, dice que «ninguna Revelación con una $\mathrm{R}$ mayúscula se puede dar en la fenomenicidad» ${ }^{5}$. A ello, agrega Marion a renglón seguido: «En pocas palabras, no tenemos experiencia de lo que rebasa las posibilidades de la experiencia; y sin embargo el pretendido fenómeno saturado, rebasa por su propia definición los límites de la experiencia» 6 .

\footnotetext{
${ }^{3}$ Jean-Luc Marion, Siendo dado, trad. Javier Basas Vila (Madrid: Editorial Síntesis, 2008), 379.

${ }^{4}$ Jean-Luc Marion, Siendo dado, 379-380. Sobre la recepción de la revelación como tema de la filosofía, Robyn Horner dice que, si la revelación es considerada dentro del dominio de la filosofía, normalmente solo aparece en su posibilidad de revelación natural o general más que como revelación especial. Y agrega que el propio Marion ha admitido que el fenómeno revelatorio a menudo ha sido excluido de su legítima consideración por parte de la filosofía y de la Universidad por su aparente falla en armonizar con el principio de razón suficiente. La revelación parece no ser un concepto que una persona racional puede tomar en cuenta de modo serio. Robyn Horner, «Revelation as a Problem for Our Age» en Jean-Luc Marion-Christiaan Jacobs Vandegeerd (editors), The Enigma of Divine Revelation. Between Phenomenology and Comparative Theology, Contributions to Hermeneutics 7 (Cham: Springer, 2020), 76. Una excelente introducción a la filosofía de Marion en su relación con la teología es la obra de Stéphane Vinolo, Jean-Luc Marion, La fenomenología de la donación como relevo de la metafísica (Quito: FCE, 2019).

5 Marlène Zeder, «Phenomenology and Trascendence» en Faulconere, James E. ed., Trascendence in Philosophy and Religion (Bloomington: Indiana UP, 2003), cap. VI, 110. Cit. por Jean-Luc Marion en «La banalidad de la saturación», Jorge L. Roggero, El fenómeno saturado. Reflexiones sobre la excedencia de la donación en la fenomenología de Jean-Luc Marion (Buenos Aires: sb Editorial, 2020), 17. También Lévinas distingue entre «revelación» y «Revelación». Dice que el contenido atribuido a la Revelación es casi metafísico, «hecho que es a su vez el primer y principal contenido revelado de toda revelación». Emmanuel Lévinas, «La Revelación en la tradición judía», en Difícil libertad, trad. Manuel Mauer (Buenos Aires: Lilmod, 2004), 417. Para Lévinas, la Revelación está contenida en el Antiguo Testamento del cual dice: «No hay un solo versículo, una sola palabra del Antiguo Testamento -leído como lectura religiosa, como Revelación- que no abra sobre todo un mundo, mundo inicialmente insospechado que envuelve lo legible», Emmanuel Lévinas, «La Revelación en la tradición judía», 420.

${ }^{6}$ Jean Luc-Marion, «La banalidad de la saturación», 17.
} 


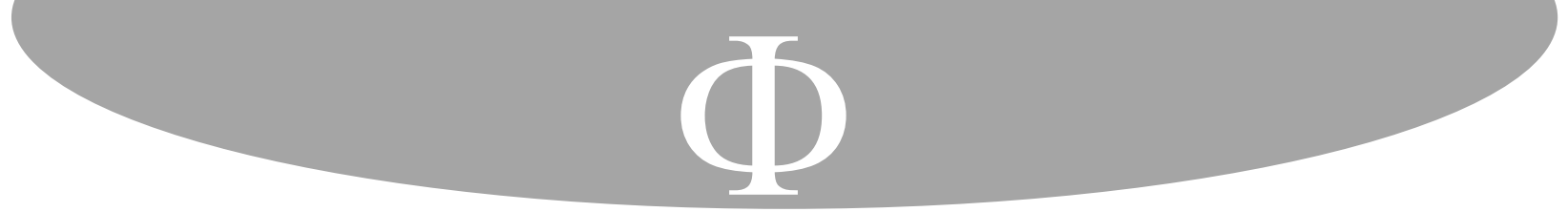

Son las que Dios ha preparado para los que le aman» ${ }^{17}$.

Quien comunica esa sophia es el Espíritu de Dios que sondea las profundidades de él (1 Co. 2.10). Según Marion, esas profundidades de Dios se corresponden con lo que el propio Jesús descubre a sus discípulos a quienes les ha sido dado a conocer el misterio del Reino de Dios (Mr. 4.11). Y afirma:

Es decir, para el mysterion de Dios, ninguna visión, ninguna interpretación, ninguna constitución es posible, a menos que sea por medio de la intencionalidad de Dios, interpretación del mismo Dios, constitución de Dios de su propio fenómeno, que puede ser visto y recibido cuando es dado. En este sentido, literalmente, el Espíritu decide y «juzga todas las cosas, anakrinei ta panta» $\left(1\right.$ Co. 2.15) ${ }^{18}$.

Este planteo de Pablo, para Marion, implica un conflicto entre «sabiduría del mundo» y la «sabiduría de Dios» (1 Co. 1.21; 2.6-7). No hay dudas que la extensa discusión que Pablo hace en 1 Co. 1.18 a 2.16 representa una oposición crítica entre la filosofía griega y la sabiduría de Dios, sophia vs. sophia.

Tal conflicto de interpretaciones resulta en una radical oposición, porque no solo representa una divergencia de opiniones o aún de tesis rivales basadas en argumentos, sino con una ruptura en la racionalidad misma, que cesa para garantizar el común espacio de comunicación, aún de una comunicación divergente. Ciertamente, esto lo hace en oposición a un logos, el Logos que muere en la cruz y desde la cual resucita (1 Co. 1.18), para «subliminar el logos o la sabiduría» (2.1) del mundo; en este sentido, se trata de razón contra razón, racionalidad contra racionalidad ${ }^{19}$.

Marion continúa con otras referencias al término logos en la perspectiva paulina. Recuerda la advertencia de no ser engañado por palabras vacías (Ef. 5.6); critica el logos de la sabiduría humana y lo contrata con el poder (dynamis) de Dios (1 Co. 2.5) porque el reino de Dios no se caracteriza por meros logoi vacíos de eficacia sino por la dynamis del Espíritu Santo (1 Co. 4.19-20 y 1 Ts. 1.5). El logos de la cruz es una verdadera locura para los que se pierden mientras que es verdadero poder de Dios para que los que se salvan (1 Co. 1.18). En ese contexto, Marion se pregunta cómo es posible comprender que «lo insensato de Dios es más sabio que los hombres» (1 Co. 1.25) y explica, tomando en cuenta a Aristóteles, que contra quien niega los principios del argumento racional, uno solo puede exponer sus contradicciones o permanecer firmemente dentro de la evidencia de la verdad. Esto, en el argumento de Pablo significa que el mundo considera como locura la «sabiduría que viene de Dios, sophia apo theou (2 Co. 1.30)» ${ }^{20}$. Hay que entender con Bultmann, que en este insondable pasaje paulino hay motivos gnósticos. El exégeta luterano explica:

\footnotetext{
171 Co. 2.9 Reina Valera, 1960. Cita de Isaías 64.4

18 Jean-Luc Marion, Givenness \& Revelation, 65. Cursivas originales.

${ }^{19}$ Jean-Luc Marion, Givenness \& Revelation, 67. Cursivas originales.

${ }^{20}$ Jean-Luc Marion, Givenness \& Revelation, 68. Cursivas originales.
} 


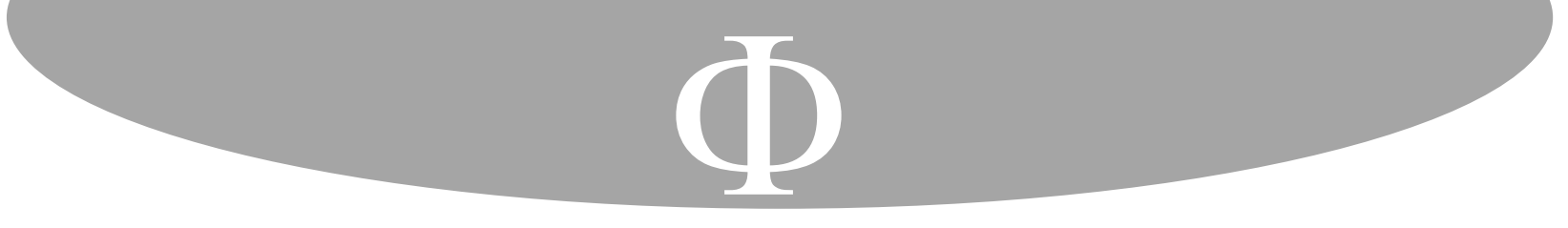

A partir de ello es que se entiende por qué Marion estima tanto el aporte de Barth a su tema: Revelación y Trinidad. Porque, para el teólogo reformado, la Revelación es precisamente el fundamento de la Trinidad ya que es el modo en que la propia Trinidad descubre (apokalipsis) el misterio de Dios articulado trinitariamente. «En una palabra, la Trinidad ofrece no sólo el contenido del descubrimiento, sino también el modo de manifestación. O, mejor dicho: el modo de manifestación (el wie fenoménico), coincide con lo que se manifiesta a sí mismo (Sich-selbst-zeigende)» ${ }^{48}$. Es decir, una armonía total entre el modo de mostración y el contenido de lo que se muestra a sí mismo.

En cuanto a la Revelación económica, Marion indica que es importante determinar de qué economía estamos hablando. Y allí discurre sobre los términos alemanes Historie y Geschichte, pero la Revelación no pertenece ni puede ser descrita por ninguno de ellos, porque la Revelación se da a través de eventos, «es decir, fenómenos saturados, que son no objetivables por medio de conceptos, y la venida de los cuales (arribo, arrivage inesperados) por lo que impone una hermenéutica ilimitada en su testimonio» ${ }^{49}$. Marion cita un texto de Gregorio Nazianceno en el que interpreta los medios en que se proclama a cada persona de la Trinidad: «El Antiguo Testamento proclamó al Padre abiertamente, y al Hijo más oscuramente. El Nuevo manifestó al Hijo, y sugirió la Deidad del Espíritu. De ahora en adelante (nyn) el Espíritu mismo habita entre nosotros, y nos suministra una más clara demostración de sí mismo» ${ }^{50}$. Respecto a la unidad como unión mediante la comunión, que evite aritmetizar la pluralidad más que la unidad, Basilio de Cesarea recalcó con claridad: «Nosotros proclamamos cada hipóstasis como única (monakhos); y, cuando contamos debemos, no hacerlo de modo que conduce a una ignorante aritmética (apaideuto arithmesen) llevándonos lejos a la idea de pluralidad de Dioses» ${ }^{51}$. Para Marion, la originalidad de la doctrina desarrollada por Basilio el Grande, descansa en su capacidad para mostrar la

\footnotetext{
48 Jean-Luc Marion, Givenness \& Revelation, 99. Cursivas originales.

49 Jean-Luc Marion, Givenness \& Revelation, 99. Cursivas originales.

${ }^{50}$ Gregorio Nacianceno, Orationes, XXXI, 26, PG 36, 161c cit. en Jean-Luc Marion, Givenness \& Revelation, 100. Cursivas originales. Con razón, Marion señala que esa división tripartita de la historia de la salvación que va del Antiguo al Nuevo Testamento es recogida después por el místico calabrés Joaquim da Fiore: reino del Padre, reino del Hijo y reino del Espíritu Santo que tanta influencia ejerció en la teología y la filosofía, entre otros, en Hegel y, más recientemente, en Gianni Vattimo. También Leonardo Boff comenta el aporte de Gregorio Nacianceno que subraya la función única del Espíritu como soplo de Dios. Dice: «En la naturaleza divina, la piedad nos obliga a creer en un Espíritu (Soplo) de Dios, ya que existe también una palabra de Dios La palabra de Dios no debe ser inferior a nuestra palabra; lo mismo que nuestra palabra viene acompañada del soplo, así la Palabra viene acompañada del Soplo (Espíritu)». Gregorio Nacianceno, Oratio cathechetica magna, 2.1, cit. por Leonardo Boff, La Trinidad, la sociedad y la liberación, 127. Según Pannenberg, contra la perspectiva arriana de Dios, Gregorio Nacianceno subrayó la incomprensibilidad de la esencia divina mientras que Gregorio Niseno se basó en la doctrina de la infinidad de Dios. Wolfhart Pannenberg, Systematic Theology vol. 1, trad. Geoffrey W. Bromiley (Grand Rapids: Eerdmans, 1998), 342.

${ }^{51}$ Basilio de Cesarea, Liber de Spiritu Sancto, XVIII, 44, PG, 32, 148a, cit en Wolfhart Pannenberg, Systematic Theology. Cursivas originales. En la misma perspectiva y, con relación al aporte general de los grandes capadocios, José Míguez Bonino destaca que ese aporte consistió en su énfasis en la comunión. -Superando al «Uno» inaccesible, los padres capadocios destacaron: «una permanente conversación, una comunión de amor, una identidad de propósito y una unidad de acción: Padre, Hijo y Espíritu Santo». José Míguez Bonino, Rostros del Protestantismo latinoamericano (Buenos Aires: Isedet-Nueva Creación, 1995), 113. Cursivas originales.
} 


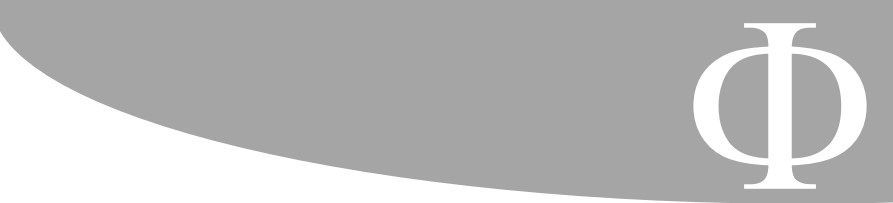

Silva, Eduardo. «La fenomenología de la donación y las (im)posibilidades de la teología». Compilado por Eric Pomier. La fenomenología de la donación. Buenos Aires: Prometeo libros, 2017.

The Greek New Testament, Third Edition. West Germany: United Bible Societies, 1976.

Vinolo, Stéphane. Jean-Luc Marion. La fenomenología de la donación como relevo de la metafísica. Quito: FCE, 2019.

Zeder, Marlène. «Phenomenology and Trascendence». Editado por Faulconere, James E. Trascendence in Philosophy and Religion. Bloomington: Indiana UP, 2003.

Zuzioulas, Ioannis D. El ser eclesial. Traducido por Francisco Javier Molina de la Torre. Salamanca: Sígueme, 2003.

Enviado: 2 de julio de 2021 Aceptado: 29 de julio de 2021 